\title{
Early and late results of surgery for Wolff-Parkinson-White syndrome
}

\author{
F. L. Rosenfeldt \\ Principal Research Fellow, Baker Medical Research Institute; Visiting \\ Assistant Surgeon, CJ Officer-Brown Cardiac Surgical Unit, Alfred \\ Hospital, VIC. \\ D. Muller \\ Cardiologist, University of Michigan Medical Centre, Ann Arbor, \\ Michigan, USA. \\ R. Harper \\ Director, Department of Cardiology, Monash Medical Centre, VIC.
}

\author{
G. C. Shardey \\ Visiting Surgeon, CJ Officer-Brown, Cardiac Surgical Unit, Alfred \\ Hospital, VIC.
}

A. Broughton

Cardiologist, Department of Cardiology, Alfred Hospital, VIC.

\begin{abstract}
:
Surgical treatment for accessory atrioventricular connections was performed in 60 patients with Wolff-Parkinson-White syndrome between 1981 and 1986. The initial procedure successfully divided 69 of 73 pathways identified preoperatively, including 39 of 40 left free wall pathways, 23 of 24 posteroseptal pathways, six of seven right free wall pathways, and one of two anteroseptal pathways. Three additional pathways were identified for the first time during follow-up. The primary procedure was successful in curing $53(88 \%)$ of the 60 patients and in dividing $69(91 \%)$ of the total of 76 pathways. Subsequent procedures increased the overall clinical cure rate to $97 \%$. The surgery was performed with low morbidity and no perioperative or late mortality. Patients were followed-up for a mean of 6.4 years (range four to nine years). No patient showed clinical or electrocardiographic evidence of recurrence of a pathway which had been divided surgically. We conclude that regardless of pathway site, surgical treatment carries a low risk and has a high probability of avoiding lifelong antiarrhythmic therapy. (Aust NZ J Med 1991; 21: 325-329.)
\end{abstract}

Key words: Arrhythmia, heart surgery, Wolff-Parkinson-White syndrome.

\section{INTRODUCTION}

Since the first successful division of an accessory atrioventricular connection by Sealy ${ }^{1}$ in 1968, surgical treatment of Wolff-Parkinson-White (W-P-W) syndrome has become an attractive alternative to lifelong medical therapy, particularly in young patients and those with the potential for life-threatening arrhythmias. The past two decades have seen major advances in the understanding of the condition and improvement in surgical techniques, which have resulted in success rates as high as $99 \% .^{2}$ We report the early results and the late outcome of the first 60 patients in whom pathway division was performed at the Alfred Hospital between February, 1981 and August, 1986.

\section{METHODS \\ Patient Population}

The 60 patients, 39 males and 21 females, aged ten to 61 years (mean 26 years), had a total of 76 pathways. Multiple pathways (two or three) were present in 14 patients $(23 \%)$. Nineteen $(25 \%)$ of the pathways were 'concealed' (no electrocardiographic delta wave). The remaining $57(75 \%)$ of the pathways manifested antegrade conduction (delta wave present).

The majority of the patients had otherwise normal

Reprint requests to: Dr F. L. Rosenfeldt, Baker Medical Research Institute, P.O. Box 348, Prahran, Vic, 3181 , Australia. Grant support: Dr Rosenfeldt's research is supported by the National Health and Medical Research Council. 
hearts. Fifteen patients had associated cardiac abnormalities or additional mechanisms for supraventricular tachycardia. Ebstein's anomaly of the tricuspid valve was present in four patients. Three of these had right free wall pathways (two with associated posteroseptal pathways) and one had a single posteroseptal pathway. Mild mitral valve prolapse was noted in four patients and an isolated ostium secundum atrial septal defect was present in one.

Dual AV nodal pathways participating in atrioventricular (AV) junctional re-entrant tachycardia were demonstrated in four patients and ectopic atrial tachycardia occurred in two patients, one in the left atrium and one in the right atrium. Concomitant surgical procedures were performed in seven of these 16 patients in addition to the ablation of one or more accessory atrioventricular connections. Tricuspid valve annuloplasty was performed in one patient with Ebstein's anomaly, AV node dissection was performed in three patients with AV node re-entry. In one patient an atrial septal defect was closed. Ectopic atrial foci were excised in two.

\section{Preoperative Evaluation}

Preoperative electrophysiological studies were performed in all patients using previously described techniques. ${ }^{3}$ Left free wall pathways were accurately located using a quadripolar coronary sinus catheter. A standard mapping catheter was used to locate the right free wall pathways. Indications for surgical management included previous cardiac arrest or potentially lethal arrhythmias (17 patients), incessant supra-ventricular tachycardia (two patients), and failed medical treatment or patient preference in the remainder. The mean duration of symptoms prior to surgery was approximately nine years.

\section{Surgical Technique}

Standard techniques of dissection described by Cox et al. ${ }^{2}$ were used for all pathways. The principle is to dissect the fat of the relevant portion of the atrioventricular groove away from the ventricle, thereby disconnecting the distal limb of the accessory atrioventricular connection.

Electrophysiological mapping was used at the time of operation to localise the connection precisely. Atrial and ventricular reference electrodes were placed, and a roving, hand-held mapping probe was used. Excitation intervals were measured using a digital timing and display unit. ${ }^{4}$ The ventricular side of the circumference of the heart at the atrio-ventricular groove was mapped during sinus rhythm, or with atrial pacing in patients with accessory pathways which conducted antegrade. The atrial side of the circumference of the atrio-ventricular free wall was mapped during reciprocating tachycardia and with ventricular pacing.
TABLE 1

Outcome of Initial Operation

\begin{tabular}{lccccc}
\hline & \multicolumn{5}{c}{ Site of pathway } \\
\cline { 2 - 6 } & LFW & PS & RFW & AS & Total \\
\hline Number present & 40 & 25 & 8 & 3 & 76 \\
Number divided & 39 & 23 & 6 & 1 & 69 \\
\% divided & 98 & 92 & 75 & 33 & 91 \\
Number mapped & 40 & 24 & 7 & 2 & 73 \\
$\%$ divided of those mapped & 98 & 96 & 88 & 50 & 95
\end{tabular}

Abbreviations: LFW-left free wall; RFW-right free wall; PS-posteroseptal; AS-anteroseptal.

Division of the accessory atrioventricular connection was performed during cardiopulmonary bypass at $28^{\circ} \mathrm{C}$. Myocardial protection was by hypothermic cardioplegia and topical cooling. For left free wall pathways, the epicardial fat space was entered through an atrial endocardial incision made $3 \mathrm{~mm}$ above the mitral annulus. Anterior and posterior septal spaces were approached through an endocardial incision above the tricuspid annulus. In the early part of the series, right free wall pathways were dissected from the epicardial surface only. With increasing experience an endocardial counter-incision was made routinely. In all cases, the epicardial fat pad was meticulously dissected away from the valve annulus and the adjacent ventricle, the limit of the dissection being the line of reflection of the epicardium.

At the completion of the procedure, after rewarming, mapping was repeated in sinus rhythm and ventricular pacing to confirm inactivation of the accessory connection and to identify any additional pathways which may have been unmasked. Temporary pacing wires were placed on the epicardial surface of the right ventricle, the right atrial appendage, the region of the interatrial septum and the left atrium. These wires were subsequently used for electrophysiology studies performed routinely seven to ten days after surgery.

\section{Postoperative Evaluation}

Following discharge from hospital, patients were reviewed three and six months after surgery and thereafter on an annual basis. The mean follow-up time was $6.4 \pm 1.6$ years (range four to nine years).

\section{RESULTS}

Seventy-three of the 76 pathways were accurately identified at the time of operation (Table 1). Of these 73, $69(95 \%)$ were successfully divided at the time of the initial surgical procedure. The four pathways which were not divided included one left free wall, one posteroseptal, one right free wall and one anteroseptal accessory pathway. Both patients, where septal pathways were missed, had dual anteroseptal and posteroseptal pathways; the anterior pathway was 
TABLE 2

Final Outcome

\begin{tabular}{lrrrrrr}
\hline & \multicolumn{5}{c}{ Site of Pathway } \\
\cline { 2 - 7 } & LFW & PS & RFW & AS & Total \\
\hline Pathways present & 40 & 25 & 8 & 3 & 76 \\
Second procedure necessary & 0 & 2 & 2 & 1 & 5 \\
Pathways ablated: & 39 & 24 & 8 & 1 & 72 \\
$\quad$ surgical division & 0 & 1 & 0 & 0 & 1 \\
$\quad$ catheter ablation & 0 & 0 & 0 & 1 & 1 \\
His bundle abiation & 98 & 100 & 100 & 66 & 97 \\
Overall success (\%) & & & &
\end{tabular}

Abbreviat:ons: LFW-teft free wall; RFW-right free wall; PS-posteroseptal: AS-anteroseptal.

missed in one and the posterior in the other. Only two of the four undivided pathways were detected at the seven day postoperative electrophysiology study. Two pathways (one left free wall and one anteroseptal) were sufficiently modified to prevent atrioventricular reentry, although both had a persistent W-P-W abnormality on the surface ECG. One of these two patients is arrhythmia free, while the other requires drug therapy to control atrioventricular junctional re-entry (AV node re-entrant tachycardia), which was not detected prior to surgery. The two remaining patients had persistent arrhythmias and required a second procedure to ablate residual pathways (Table 2). A right free wall pathway was successfully divided surgically in one patient and, in the second, a posteroseptal pathway was successfully treated by percutaneous catheter electrode ablation.

Three of the 76 pathways were AV connections which were undetected both at initial and intraoperative electrophysiology studies. These included one posteroseptal, one anteroseptal and one right free wall pathway. All three pathways were detected before discharge from hospital at the routine postoperative electrophysiology study and subsequently confirmed by detailed electrophysiology study. All three patients presented one to seven months postoperatively with recurrent supraventricular tachycardia. One patient subsequently underwent a successful posteroseptal dissection. A second patient underwent surgical ablation of the His bundle and insertion of a permanent ventricular pacemaker. Both patients remain asymptomatic without therapy after five and eight years respectively. The third patient, a young man with Ebstein's anomaly, had postoperative supraventricular tachycardia following successful division of a right free wall (using the epicardial approach alone) and a posteroseptal pathway. A subsequent electrophysiology study identified a second right free wall pathway and dual AV nodal pathways and previously unsuspected AV junction re-entry. A second surgical procedure, utilising a combined epicardial-endocardial dissection and endocardial cryolesions, successfully ablated the SURGERY FOR W-P-W SYNDROME residual accessory pathway and abolished retrograde conduction through the AV node.

Despite this, supraventricular tachycardia recurred. Findings at a third electrophysiology study were consistent with intranodal re-entry with complete atrial dissociation. ${ }^{5}$ Abolition of the arrhythmia was finally achieved by catheter ablation of the His bundle and permanent atrioventricular sequential pacing.

\section{Complications}

The surgery was performed with no perioperative or late mortality and few complications. Four patients, each following a left free wall dissection, had transient postoperative hypotension requiring inotropic support for periods of 24 to 28 hours. Subsequent assessment showed no lasting deterioration in ventricular function compared with pre-operative levels. Transient complete heart block occurred in four patients, including two of three undergoing a combined free wall and AV nodal dissection. In each of these four patients, $1: 1 \mathrm{AV}$ conduction returned within 48 hours and none has had conduction problems in the long term. Two patients developed haemodynamically significant pericardial effusions which required surgical drainage during the late postoperative period. This incidence (3\%) of post-cardiotomy pericardial effusions is comparable with that following other cardiac surgical procedures. Paroxysmal atrial fibrillation persisted beyond the early post-operative period in one patient only and resolved within six months. No patient developed new atrial arrhythmias after discharge from hospital. After discharge no patient showed clinical or electrocardiographic evidence of recurrence of a pathway which had been successfully divided.

\section{DISCUSSION}

We report the first 60 patients undergoing surgical treatment for W-P-W syndrome at the Alfred Hospital, with a high success rate in the medium and long term, low morbidity and zero mortality. Overall, the potential for atrioventricular re-entrant tachycardia was completely abolished in 58 of the 60 patients, including the five patients who had a second procedure. Of the remaining two patients, one is symptom-free despite the presence of a residual accessory atrioventricular connection. The other patient requires ongoing antiarrhythmic therapy for intermittent atrioventricular junctional re-entrant tachycardia which was not recognised preoperatively. This patient is the only one in the series requiring continuing medical treatment.

Recent advances in the accuracy of pre- and intraoperative electrophysiology studies have allowed curative surgery to become an attractive option for patients with W-P-W syndrome. Of the numerous surgical techniques which have been devised to ablate the accessory connection, the endocardial approach ${ }^{2}$

Aust NZ J Med 1991; 21 
is perhaps the most widely employed. Alternative techniques include cryoablation ${ }^{6}$ or epicardial dissection, ${ }^{7}$ each of which avoids the need for aortic cross-clamping and hypothermic cardioplegic arrest. The success rates for endocardial and epicardial dissection do not seem to be significantly different, but in some series the latter has been associated with an unacceptably high incidence of haemorrhagic complications. ${ }^{7}$

Uther et al. reported a relatively low success rate for surgical division of septal pathways. ${ }^{8}$ In the present series, with the possible exception of anteroseptal pathways, where the numbers were small, pathway site had little influence on success rate. The main factor limiting success was the adequacy of identification of multiple pathways or multiple mechanisms of tachycardia. Additional pathways were unrecognised in two of $14(14 \%)$ patients with multiple accessory AV connections and one of four patients with both an accessory pathway and AV node re-entry. Multiple pathways should be suspected in all patients with W$\mathrm{P}$-W syndrome, but especially in those with associated Ebstein's anomaly, because the combination of right free wall and posteroseptal pathways is particularly common in this condition.

Long term complications following surgical division of accessory pathways appear to be negligible, although few centres have follow-up periods beyond ten years. Once surgical division has been successfully accomplished, recurrence of re-entrant tachycardia after one year is rare. ${ }^{2}$ Likewise in the subset of patients with W-P-W syndrome who are prone to atrial fibrillation, Sharma et al. noted a marked reduction in the incidence of atrial fibrillation during a mean postoperative follow-up period of two years. ${ }^{9}$ They postulated that AV re-entry is responsible for the vulnerability of the atrium to fibrillation and that the accessory pathway may be required for its initiation. If such a mechanism is responsible for the increased incidence of atrial fibrillation in patients with accessory AV connections, it might be expected that surgically treated patients would remain free of all atrial arrhythmias once the pathway has been effectively ablated.

Although less invasive procedures such as catheter ablation and antitachycardia pacemakers have recently been advocated, surgical division remains the procedure with the highest success rate and the lowest morbidity. It should therefore still be considered the procedure of choice for patients with W-P-W syndrome and arrhythmias which are life-threatening or refractory to medical therapy. Even in patients with non life-threatening arrhythmias controlled by medical therapy, surgical division of pathways is attractive because it avoids the inconvenience, cost and side effects of long term drug therapy.

Catheter electrode ablation using DC electric shocks has been used to ablate some accessory pathways. ${ }^{10-13}$
Currently the technique is mainly reserved for posteroseptal accessory pathways with the electric shock being delivered just outside or just within the orifice of the coronary sinus. So far only some small series with short follow-up times have been reported with success rates in the order of $70 \%,{ }^{11-13}$ and a significant incidence of serious complications including AV block, myocardial infarction, coronary sinus rupture, pericardial tamponade and death. One group ${ }^{12}$ advises that the procedure be performed in an operating theatre with provision for immediate cardiac surgery should coronary sinus rupture and tamponade occur. Left free wall pathways, which are the commonest site for accessory pathways, are currently not amenable to catheter electrode electrical ablation from the coronary sinus because of the high likelihood of coronary sinus rupture. ${ }^{10}$ The results of electrical ablation of right free wall accessory pathways have in general been disappointing. Future modifications of the catheter electrode technique including the use of radiofrequency energy, ${ }^{14}$ may allow more patients with accessory pathways to be treated in this way, but in our opinion, surgical treatment is still the method of choice for definitive correction of the W-P-W syndrome.

\section{Addendum}

As of August 31 1990, a total of 113 patients at the Alfred Hospital had surgery for W-P.W Syndrome, without mortality.

\section{Acknowledgements}

The authors acknowledge the advice and assistance provided, in the early stages of the arrhythmia surgery programme at the Alfred Hospital, by Dr J. Uther and Mr D. Johnson from Westmead Hospital, Sydney.

Accepted for publication: 11 March 1991.

\section{References}

1. Cobb FR, Blumenschein SD, Sealy WC, Boineau LP, Wagner GS, Wallace AG. Successful surgical interruption of the bundle of Kent in a patient with Wolf-Parkinson-White syndrome. Circulation 1968; 38: 1018-29.

2. Cox JL, Gallagher JJ, Cain ME. Experience with 118 consecutive patients undergoing operation for the Wolff-Parkinson-White syndrome. J Thorac Cardiovasc Surg 1985; 90: 490-501.

3. Harper RW, Whitford E, Middlebrook K, Federman J, Anderson S, Pitt A. Effects of verapamil on the electrophysiologic properties of the accessory pathway in patients with the Wolff-Parkinson-White syndrome. Am J Cardiol 1968; 50: 132330.

4. Rosenfeldt FL, Harper RW, Wall RE, Uther JB, Hilder R, Shardey GC. A digital timing and display unit for intraoperative mapping of cardiac arrhythmias. PACE 1984; 7: 985-92.

5. Harper RW, Broughton A, Weissberg P, Shardey G, Muller $D$, Pitt A. Paroxysmal atrioventricular junctional re-entrant tachycardia with complete atrial dissociation following unsuccessful surgery for the condition (Abstr.). Aust NZ J Med 1987; 17 Suppl. 4: 577.

6. Camm J, Ward DE, Spurrell RAJ, Rees GM. Cryothermal mapping and cryoablation in the treatment of refractory cardiac arrhythmias. Circulation 1980; 62: 67-74. 
7. Guiraudon GM, Klein GJ, Sharma AD, Jones DL, McLellan DG. Surgery for Wolff-Parkinson-White syndrome: further experience with an epicardial approach. Circulation 1986; 74: 525-9.

8. Uther JB, Johnson DC, Baird DK $e_{t}$ al. Surgical section of accessory atrioventricular electrical connections in 108 patients (Abstr.). Am J Cardiol 1982; 49: 995.

9. Sharma AD, Klein GJ, Guiraudon GM, Milstein S. Atrial fibrillation in patients with Wolff-Parkinson-White syndrome: incidence after surgical ablation of the accessory pathway. Circulation 1985; 72: 161-9.

10. Fisher JD, Brodman R, Kim SG, Matos JA et al. Attempted nonsurgical electrical ablation of accessory pathways via the coronary sinus in the Wolff-Parkinson-White syndrome. J Am Coll
Cardiol 1984; $4: 685-94$

11. Morady F, Scheinman MM, Winston SA et al. Efficacy and safety of transcatheter ablation of posteroseptal accessory pathways. Circulation 1985; 72: 170-7.

12. Bardy GH, Ivey TD, Coltorti F, Stewart RB, Johnson G, Greene HL. Developments, complications and limitations of cathetermediated electrical ablation of posterior accessory atrioventricular pathways. Am J Cardiol 1988; 61: 309-16.

13. Evans GT Jr, Scheinman MM, Zipes DP et al. The Percutaneous Cardiac Mapping and Ablation Registry: final summary of results. PACE 1988; 11: 1621-4.

14. Jackman W, Wang X, Moulton $\mathrm{K}$ et al. Role of the coronary sinus in radiofrequency ablation of left free-wall accessory $A V$ pathways (Abstr.). Circulation 1990; 82 Suppl. III-719.

\section{Fast Tracking Process - information for contributors}

C ontributors are reminded that the Journal offers rapid publication for selected articles in a special supplement which will appear after the advertisements in the Journal each month but will be continuous as far as page numbering goes.

A uthors are invited to nominate papers for rapid publication and, if their recommendation meets with the agreement of the sub-editor in the field, the paper will be refereed to allow acceptance or rejection within five weeks and publication in the next issue of the Journal provided we are left with a three weeks printing time. It is intended that authors with important data for which they wish to establish precedence will be able to do so in the Australian and New Zealand Journal of Medicine. 